Pacific Journal of Mathematics

ON THE CONVERGENCE OF SEMI-DISCRETE ANALYTIC

G. J. KURowsk 


\section{ON THE CONVERGENCE OF SEMI-DISCRETE ANALYTIC FUNCTIONS}

\section{G. J. KuRowskI}

1. Introduction. In a previous paper [3], the author has presented the basic concepts and definitions for semi-discrete analytic functions. These functions are defined on two types of semi-lattices (sets of lines in the $x y$-plane, parallel to the $x$-axis)-one of which leads to a symmetric theory. We will concern ourselves here only with the symmetric case. These functions satisfy the following defining equation [3] on a region of the semi-lattice

$$
\frac{\partial f(z)}{\partial x}=[f(z+i h / 2)-f(z-i h / 2)] / i h,
$$

where $h>0$ is the spacing of the semi-lattice. For convenience, we will repeat the definition of the symmetric semi-lattice and its associated odd and even semi-lattices. A grid-line, $a_{m}$, is the set of points in the $x y$-plane such that $y=m h$ where $h>0$. The union $G(2 k, h)$ of the $a_{m}$ for $m=k(k=0, \pm 1, \pm 2, \cdots)$ is called the even semi-lattice; the union $G(2 k+1, h)$ of the $a_{m}$ for $m=(2 k+1) / 2$ is called the odd semi-lattice. The semi-discrete $z$-plane is the union of $G(2 k, h)$ and $G(2 k+1, h)$. It will be denoted by $L(h)$. Additional concepts such as domains, paths, path-integrals, etc., are defined in [3]. The following notational conventions will be employed:

$$
f_{k}=f(x+i h k)=f_{k}(x),
$$

and the abbreviation $S D$ will be used to stand for semi-discrete.

2. Sub and super harmonic semi-discrete functions. In the continuous case, it is well-known that if a function $u(x, y)$ is defined over a region $R$ of the plane and if, further, $\Delta^{2}(u) \geqq 0$ for all $(x, y) \in R$, where $\Delta^{2}$ denotes the two dimensional Laplacian; then $u(x, y)$ cannot have a maximum on the interior of $R$. Such a function is said to be sub-harmonic in $R$ [2]. Similarly, if the function $u(x, y)$ defined on $R$ satisfies the equation $\Delta^{2}(u) \leqq 0$ for all $(x, y) \in R$; then $u(x, y)$ cannot have a minimum on the interior of $R$. Such a function is said to be super-harmonic in $R$ [2]. An analogous result holds for semi-discrete functions which are defined on domains of either the even or odd semilattice. To be specific, we will consider functions $u(x, y)$ defined on

Received April 17, 1963. Duke University Research Associate, "Special Research in Numerical Analysis," sponsored by the Army Research Office (Durham), U.S. Army, Contract DA-31-124-AROD-13. 
domains of $G(2 k, h)$ and introduce the notation

$$
\begin{aligned}
& \text { (a) } h E u(x, y)=u(x, y+h)-u(x, y) \text {, } \\
& \text { (b) } h \bar{E} u(x, y)=u(x, y)-u(x, y-h) .
\end{aligned}
$$

The semi-discrete Laplacian operators for $G(2 k)$ is then

$$
\nabla u(x, y)=\frac{\partial^{2} u(x, y)}{\partial x^{2}}+E \bar{E} u(x, y) \text {. }
$$

THeOREM 2.1. Let $u(x, y)$ be a SD-function defined on a semidiscrete domain $R$ of $G(2 k, h)$. If $\nabla u \geqq 0$ for all $(x, y) \in R$, then on $R$

$$
u(x, y) \leqq M,
$$

where $M$ is the supremum of $u(x, y)$ on $C$, the boundary of $R$.

Proof. The proof of this statement is obtained by a suitable modification of the proof for the "weak maximum theorem" established by Helmbold [1] for semi-discrete harmonic functions. Let $C$ denote the boundary of the SD-domain $R$ of $G(2 k, h)$, let $u(x, y)$ be a SDfunction on $R$ such that $\nabla u \geqq 0$ for all $(x, y) \in R$, and let $M^{\prime}$ denote the supremum of $u(x, y)$ on $R$. Assume that $u$ takes the value $M^{\prime}$ at a point $(t, n h)$ of the interior $R^{0}=R \sim C$ of $R$. If the adjacent points $(t,(n \pm 1) h)$ are points of $R^{0}, \partial^{2} u / \partial x^{2}=u^{\prime \prime}$ will be continuous at $(t, n h)$ and further $u_{n}^{\prime \prime}(t) \leqq 0$. By assumption $\nabla u_{n}(t) \geqq 0$ which, together with the previous remarks, implies that

$$
u_{n}(t)=u_{n+1}(t)=u_{n-1}(t)=M^{\prime} .
$$

This argument may be repeated for the sequence of points $(t,(n \pm 1) h)$, $(t,(n \pm 2) h), \cdots$ until a point $(t, p h)$ is reached such that one of its adjacent points is a point of $C$. If $u_{p}^{\prime \prime}$ is continuous, the proof is complete. Otherwise, since $u_{p}^{\prime \prime}$ is then at least piecewise continuous, integration of $\nabla u_{p} \geqq 0$ shows that for some range of values of $\varepsilon>0$

$$
u_{p}^{\prime}(t+\varepsilon)-u_{p}^{\prime}(t) \geqq \varepsilon h^{-2}\left\{2 u_{p}(\theta)-u_{p+1}(\theta)-u_{p-1}(\theta)\right\},
$$

where $t \leqq \theta \leqq t+\varepsilon$. Since $u_{p}=M^{\prime}$ is a maximum, the left side of (b) is negative. Hence, the bracketed term is negative. Taking the limit of this term as $\varepsilon \rightarrow 0, \varepsilon>0$ shows that

$$
2 M^{\prime} \leqq u_{p+1}\left(t^{+}\right)+u_{p-1}\left(t^{+}\right) \text {. }
$$

Similarly, we obtain

$$
2 M^{\prime} \leqq u_{p+1}\left(t^{-}\right)+u_{p-1}\left(t^{-}\right) .
$$

Addition of (c) and (d) shows that $M^{\prime} \leqq M$ where $M$ is the maximum 
value of $u(x, y)$ on $C$.

In an identical manner, we establish the following result for super SD-harmonic functions.

THEOREM 2.2. Let $u(x, y)$ be a SD-function defined on a semidiscrete domain $R$ of $G(2 k, h)$. If $\nabla u \leqq 0$ for all $(x, y) \in R$, then on $R$

$$
u(x, y) \geqq m,
$$

where $m$ is the infimum of $u(x, y)$ on $C$, the boundary of $R$.

3. Limit theorem for semi-discrete analytic functions. A SDfunction $f(z)$ of the complex variable $z=x+i n h$ which is continuous and single-valued on a SD-domain $R$ of $L(h)$ is said to be SD-analytic if it satisfies (1.1) for all points $z \in R$ [3]. In addition, if we write $f=u+i v$, then $\nabla u=\nabla v=0$ on $R$; that is, $u$ and $v$ are SD-harmonic.

Let us suppose that $L(h)$ is superimposed upon the continuous $z$ plane, denoted by $L_{c}$, with their $x$ and $y$ axes coinciding. Let $R_{c}$ be a simply-connected finite domain of $L_{c}$ whose boundary is a Jordan curve. A covering set of rectangles, $Q_{k}$, is defined as follows,

$$
Q_{k}=\left\{(x, y): \alpha_{k} \leqq x \leqq \beta_{k} ;(k h-h) \leqq 2 y \leqq(k h+h)\right\},
$$

where $\alpha_{k}$ is the least value of $x$ in $R$ taken on the strip $k h-h \leqq$ $2 y \leqq k h+h$, and $\beta_{k}$ is the greatest value of $x$ in $R$ on this strip. By construction, each point of $R_{c}$ is also a point of $Q=\bigcup_{k} Q_{k}$. The intersection of $Q$ with $L(h)$ forms a SD-domain, $R(h)$, which approximates $R_{c}$. We consider the sequence of SD-domains $\left\{R\left(h_{j}\right) ; h_{1}>h_{2}>\cdots\right\}$ obtained by the above procedure upon successive refinements of the semi-lattice retaining at each step the lines of the previous semilattice. In the limit, $R\left(h_{j}\right) \rightarrow R_{c}$. It is shown in [3] that a SDanalytic function is completely determined in $R(h)$ by its values on $C(h)$, the total-boundary of $R(h)$. Therefore, let us assume that an interpolation scheme is established to provide such boundary values for a SD-analytic function $f^{(h)}(z)$ on $R(h)$ from the boundary values of an analytic function $\zeta(z)$ on $R_{c}$ such that these approximate boundary values tend uniformly to the true boundary values. We consider the sequence of SD-analytic functions $\left\{f^{\left(h_{j}\right)}(z)\right\}$ so determined on $\left\{R\left(h_{j}\right)\right\}$ and will prove that as $h_{j} \rightarrow 0, f^{\left(h_{j}\right)}(z) \rightarrow \zeta(z)$.

THEOREM 3.1. Let $R$ be a domain whose boundary $C$ is a Jordan curve and let $R^{\prime}$ be a subdomain of $R$ which is bounded by a Jordan curve $C^{\prime} \subset R$. Consider the set of all possible semi-lattices $G(2 k, h)$ parallel to the real axis of the z-plane. Consider also the set of all SD-functions $u^{(h)}(x, y)$ which are uniformly bounded, $|u| \leqq M$ in $R$, 
and which satisfy in $R$ the equation $\nabla u=0$. Then, for $h$ sufficiently small, there exists a constant $M^{\prime}$ such that

$$
\left|\frac{\partial u^{(h)}}{\partial x}\right| \leqq M^{\prime} \quad \text { and } \quad\left|\nabla u^{(h)}\right| \leqq M^{\prime}
$$

for all $(x, y) \in R$.

Proof. The proof of this statement follows the proof given by Fellow [4] for the discrete case. The sub-domain $R^{\prime}$ can be covered by a finite number of rectangles contained in $R$ and each of these rectangles can be inclosed in a larger rectangle also contained in $R$. Following the argument of Feller [4], it will be sufficient to consider, for an arbitrary $\delta>0$, the two concentric rectangles

$$
\begin{aligned}
R & =\{(x, y):|x|<a-\delta,|y|<b\} \\
R^{\prime} & =\{(x, y):|x|<a-\delta,|y|<b-\delta / 3\},
\end{aligned}
$$

where $b$ is a multiple of the gap $h$, and $h<\delta / 3$.

To prove the assertion, we shall show that the function

$$
\psi(x, y)=\left(\frac{\partial u}{\partial x}\right)^{2} \Phi(x, y)+C\left\{u^{2}(x, y)+u^{2}(x, y+h)+u^{2}(x, y-h)\right\}
$$

where $\Phi(x, y)=\left(x^{2}-a^{2}\right)^{2}\left(y^{2}-b^{2}\right)^{2}$ and $C$ is a large positive constant, to be determined later, satisfies the inequality $\nabla(\psi) \geqq 0$.

Assume for the moment that this has been established. Then, by Theorem 2.1, it follows that $\psi$ attains its maximum value on the boundary. However, by definition, $\Phi=0$ on the boundary and thus in the entire rectangle

$$
0 \leqq \psi(P) \leqq 3 C M^{2}
$$

where $M$ is the uniform bound on $u$. Since the second term of $\psi$ is nonnegative, we may conclude that for all $P \in R^{\prime}$

$$
\left(\frac{\partial u}{\partial x}\right)^{2} \leqq 3 C M^{2} / \Phi \leqq 3 C M^{2} /(\delta / 3)^{8}
$$

[since for small $\left.\delta, \Phi \geqq(\delta / 2)^{4}(\delta / 3)^{4} \geqq(\delta / 3)^{8}\right]$.

Since $(\delta / 3)^{8}>0$, taking the last expression for $M^{\prime}$ establishes the theorem, subject to showing that $\nabla(\psi) \geqq 0$. Only the outline of this calculation will be presented. The complete sequence of steps follows the argument given by Feller [4] using the differential rather than the difference operator on $x$.

Calculation of $\nabla \psi$ using the fact that $u$ is SD-harmonic [as is $u^{\prime}$ ] gives 
(a)

$$
\begin{aligned}
\nabla(\psi)= & \left(u^{\prime}\right)^{2} \nabla(\Phi)+\Phi\left[2\left(u^{\prime \prime}\right)^{2}+\left(E u^{\prime}\right)^{2}+\left(\bar{E} u^{\prime}\right)^{2}\right] \\
& +\Phi^{\prime}\left(4 u^{\prime} u^{\prime \prime}\right)+E \Phi\left[u_{1}^{\prime} E u^{\prime}+u^{\prime} E u^{\prime}\right] \\
& +\bar{E} \Phi\left[u_{-1}^{\prime} \bar{E} u^{\prime}+u^{\prime} \bar{E} u^{\prime}\right]+C\left[2\left(u^{\prime}\right)^{2}+(E u)^{2}+(\bar{E} u)^{2}\right] \\
& +C\left[2\left(u_{1}^{\prime}\right)^{2}+\left(E u_{1}\right)^{2}+\left(\bar{E} u_{1}\right)^{2}+2\left(u_{-1}^{\prime}\right)^{2}+\left(E u_{-1}\right)^{2}+\left(\bar{E} u_{-1}\right)^{2}\right]
\end{aligned}
$$

where $u_{ \pm 1}=u(x, y \pm h)$. Since $|\partial \Phi / \partial x|=4\left|x\left(y^{2}-b^{2}\right)\right| \sqrt{\Phi}$, a constant $\lambda$ exists such that for all points of $R\left|\Phi^{\prime}\right|<\lambda \sqrt{\Phi}$. Similar bounds exist for $E \Phi$ and $\bar{E} \Phi$. Further, in $R, \nabla(\Phi)$ is bounded. Accordingly we assume that $\lambda$ is so chosen that on $R$

$$
|\nabla(\Phi)|<\lambda, \quad\left|\Phi^{\prime}\right|<\lambda \sqrt{\Phi}, \quad|E \Phi|<\lambda \sqrt{\Phi}, \quad|\bar{E} \Phi|<\lambda \sqrt{\Phi} .
$$

For an arbitrary $\varepsilon>0$, we see that

$$
\left|u^{\prime} u^{\prime \prime} \Phi^{\prime}\right| \leqq\left(\frac{u^{\prime}}{\varepsilon}\right)^{2}+\varepsilon^{2} \lambda^{2} \Phi\left(u^{\prime \prime}\right)^{2} .
$$

With such bounds established for the various terms which appear in (a), the following inequality is obtained.

$$
\begin{aligned}
\nabla(\psi) \geqq & {\left[\left(E u^{\prime}\right)^{2}+\left(\bar{E} u^{\prime}\right)^{2}+2\left(u^{\prime \prime}\right)^{2}\right] \Phi\left(1-2 \varepsilon^{2} \lambda^{2}\right) } \\
& +2\left(u^{\prime}\right)^{2}\left[C-3 / \varepsilon^{2}\right]+C\left[(E u)^{2}+(\bar{E} u)^{2}+\left(E u_{1}\right)^{2}\right] \\
& +C\left[\left(\bar{E} u_{1}\right)^{2}+\left(E u_{-1}\right)^{2}+\left(\bar{E} u_{-1}\right)^{2}\right]+\left(u_{1}^{\prime}\right)^{2}\left[2 C-1 / \varepsilon^{2}\right] \\
& +\left(u_{-1}^{\prime}\right)^{2}\left[2 C-1 / \varepsilon^{2}\right] .
\end{aligned}
$$

Selecting $\varepsilon$ so that $\varepsilon^{2} \lambda^{2}=1 / 2$, the first term on the right in (b) vanishes. Finally, choosing $C \geqq 3 / \varepsilon^{2}$, the remaining terms on the right in (b) will be positive. That is, $\nabla(\psi) \geqq 0$.

THEOREM 3.2. Let $\left\{u^{(h)}(x, y)\right\}$ be the set of uniformly bounded SD-functions considered in Theorem 3.1. This set is a family of equi-continuous functions on $R$.

Proof. In Theorem 3.1 we established the existence of a uniform bound for the set $\left\{\partial u^{(h)} / \partial x\right\}$ and also $\left\{E u^{(h)}\right\}$. Let $M$ denote this bound. (1) Given $\varepsilon>0$, let $P, Q$ be two points on a line of the semi-lattice such that $\overline{P Q}<\varepsilon / M$; that is, $\left|x_{P}-x_{Q}\right|<\varepsilon / M$, where $x_{P}$ denotes the $x$-coordinate of $P$ and $x_{Q}$ denotes the $x$-coordinate of $Q$. Then

$$
\left|u^{(h)}(P)-u^{(h)}(Q)\right|=\left|\int_{x_{Q}}^{x_{P}} \frac{\partial u^{(h)}}{\partial t} d t\right| \leqq\left[M^{2}\left(x_{P}-x_{Q}\right)^{2}\right]^{1 / 2} \leqq \varepsilon .
$$

(2) Given $\varepsilon>0$, let $P, Q$ be two points of $R$ which lie on a vertical line in $R$ such that $\left|y_{P}-y_{Q}\right|<\varepsilon / M h$.

$$
\left|u^{(h)}(P)-u^{(h)}(Q)\right|=h\left|\sum_{y=y_{Q}}^{y=y_{P}} E u^{(h)}\right| \text {. }
$$


Thus,

$$
\left|u^{(h)}(P)-u^{(h)}(Q)\right| \leqq\left|y_{P}-y_{Q}\right| M h \leqq \varepsilon .
$$

(3) Given $\varepsilon>0$, let $P, Q$ be two arbitrary points of $R$ such that $\overline{P Q}<\delta(\varepsilon)$. Let $P^{\prime}$ lie on the same vertical line as $P$ and have the same $y$-coordinate as $Q$; i.e., $P^{\prime}=\left(x_{P}, y_{Q}\right)$. Then

$$
\left|u^{(h)}(P)-u^{(h)}(Q)\right| \leqq\left|u^{(h)}(P)-u^{(h)}\left(P^{\prime}\right)\right|+\left|u^{(h)}\left(P^{\prime}\right)-u^{(h)}(Q)\right| .
$$

Application of the two previous cases completes the proof.

By Theorem 3.2, if $\left\{f^{(h)}=u^{(h)}+i v^{(h)}\right\}$ is a set of uniformly bounded SDA functions, this set is a family of equicontinuous functions which, by Kellogg [2], contains a subsequence converging uniformly in $R^{\prime}$ to a continuous limit. Since $R^{\prime}$ was an arbitrary closed sub-domain of $R$, we can choose a sequence of such regions $R^{\prime} \subset R^{\prime \prime} \subset \cdots \subset R$ whose sum is $R$ and find successive subsequences of $f^{\left(h_{1}\right)}, f^{\left(h_{2}\right)}, \ldots$ which converge in each of these regions to a continuous function. The resultant diagonal subsequence will converge uniformly to a continuous function in all of $R$. Since successive differences and derivatives of SD-harmonic functions are again SD-harmonic, the arguments in Theorems 3.1 and 3.2 can be repeated to show that there is a subsequence of the final subsequence whose first derivative and first difference ratio also converge in $R$. Thus, we can find a final subsequence which will have an arbitrary number of successive derivatives or differences which converge in $R$. Denote this final convergent subsequence by $\left\{f_{*}^{(h)}\right\}$ and let $\zeta(z)$ be the continuous function in $R$ to which it converges.

Let $C$ be a rectifiable curve in $L_{c}$. By the construction of $Q$, each point of $C$ is a point of $Q$. Consider a rectangle $Q_{k}$ of $Q$ which contains a segment $C_{k}$ of $C$. To be explicit, we will assume that $C_{k}$ intersects $Q_{k} \cap L(h)$ at the three points $p_{1}=\left(x_{1}, h(k-1) / 2\right), p_{2}=$ $\left(x_{2}, h k / 2\right)$, and $p_{3}=\left(x_{3}, h(k+1) / 2\right)$, and that the positive direction is from $p_{1}$ to $p_{3}$. The remaining possibilities can be treated by suitable modifications of the following discussion. On $Q_{k} \cap L(h)$, three SDpaths may be defined. The upper SD-path consists of the points $p_{1}$, $\left(x_{1}, h k / 2\right)$, and the line segment from $x_{1}$ to $x_{3}$ with $y=h(k+1) / 2$. The lower SD-path is the line segment from $x_{1}$ to $x_{3}$ with $y=h(k-1) / 2$, the points $\left(x_{3}, h k / 2\right)$, and $p_{3}$. The mixed SD-path consists of the line segment from $x_{1}$ to $x_{2}$ with $y=h(k-1) / 2$, the point $p_{2}$, and the line segment from $x_{2}$ to $x_{3}$ with $y=h(k+1) / 2$. At least one of these SD-paths must lie within $R(h)$ and will be chosen to be the SD approximation of the segment $C_{k}$. The SD-Cauchy theorem [3] shows that it is immaterial which SD-path is chosen if more than one of these approximating SD-paths lies within $R(h)$. The SD-path on $R(h)$ which approximates $C$ is the union of the SD-paths chosen to approximate its segments, $C_{k}$. 
THEOREM 3.3. Let $\zeta(z)$ be a continuous function on a domain $R$ and let $C$ be a rectifiable [or Jordan] curve which is contained in $R$. If $C_{h}$ is a SD-path contained in $R_{h}$ which approximates $C$, then

$$
\lim _{h \rightarrow 0} \int_{c_{h}} \zeta(z) \delta z=\int_{0} \zeta(z) d z
$$

Proof. By the definition for SD-path integration [3],

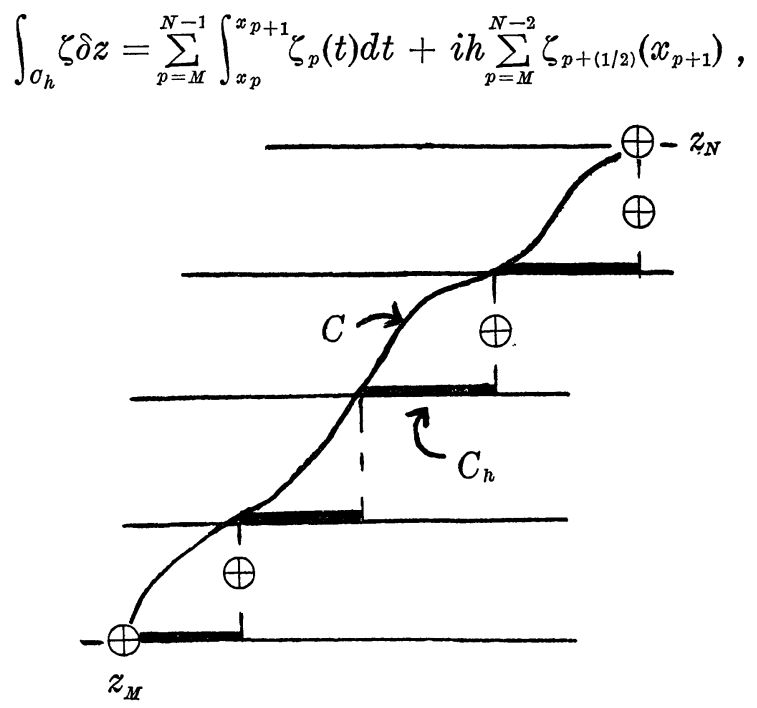

where $C_{h}$ is a SD-path joining $z_{M}=x_{M}+i M$ and $z_{N}=x_{N}+i N$. We note that as $h \rightarrow 0$, so must $\left|x_{p}-x_{p+1}\right| \rightarrow 0$. Since $\zeta$ is continuous, there exists a value $\lambda_{p}$ where $x_{p} \leqq \lambda_{p} \leqq x_{p+1}$ such that

$$
\int_{o_{h}} \zeta \delta z=\sum_{p=M}^{N-1}\left[x_{p+1}-x_{p}\right] \zeta\left(\lambda_{p}\right)+i h \sum_{p=M}^{N-2} \zeta_{p+(1 / 2)}\left(x_{p+1}\right) .
$$

As $h \rightarrow 0$ the right side of the above converges to the value of the path-integral of the continuous function $\zeta$ along the path $C$.

THEOREM 3.4. Let $R\left(h_{k}\right)$ denote a sequence of semi-lattices on a domain $R$ such that $h_{k} \rightarrow 0$, and let $f^{\left(h_{k}\right)}$ be semi-discrete analytic on $R\left(h_{k}\right)$. If the collection of these $f^{\left(h_{k}\right)}$ is uniformly bounded in $R$, then it contains a subsequence that converges everywhere in $R$ to a function $\zeta(z)$ that is analytic in $R$.

Proof. This subsequence is the final subsequence obtained in the previous discussion. Let $C$ denote an arbitrary closed rectifiable path in $R$ and let $C_{h}$ be a closed SD-path on $R\left(h_{k}\right)$ which approximates $C$. Then 


$$
\lim _{h \rightarrow 0} \oint_{\sigma_{h}} f_{*}^{\left(h_{l}\right)} \delta z=\oint_{\sigma} \zeta(z) d z,
$$

where $\left\{f_{*}^{\left(h_{k}\right)}\right\}$ is the subsequence which converges to $\zeta$. To establish (a) we consider

$$
\left|\oint_{\sigma_{h}} f_{*}^{\left(h_{k}\right)} \delta z-\oint_{\sigma} \zeta(z) d z\right| \leqq\left|\oint_{\sigma_{h}}\left(f_{*}^{\left(h_{k}\right)}-\zeta\right) \delta z\right|+\left|\oint_{\sigma_{h}} \zeta \delta z-\oint_{\sigma} \zeta d z\right| \cdot
$$

Since $f_{*}^{\left(h_{k}\right)} \rightarrow \zeta$, given $\varepsilon>0$ there exists $\delta_{1}(\varepsilon)>0$ such that the first term on the right side of (b) is smaller than $\varepsilon / 2$ provided $h_{k}<\delta_{1}$. Similarly by Theorem 3.3 , there exists $\delta_{2}(\varepsilon)>0$ such that the second term on the right side of (b) is smaller than $\varepsilon / 2$ provided $h_{k}<\delta_{2}$. Thus, on letting $\delta=\max \left(\delta_{1}, \delta_{2}\right)$

$$
\left|\oint_{O_{h}} f_{*}^{\left(h_{k}\right)} \delta z-\oint_{0} \zeta d z\right|<\varepsilon,
$$

provided $h_{k}<\delta$. This establishes (a). However, since $f_{*}^{\left(h_{k}\right)}$ is SDA for each $h_{k}$, the left side of (a) is always zero. Thus

$$
\oint_{o} \zeta(z) d z=0 \text {. }
$$

Since $C$ is an arbitrary closed rectifiable curve of $R$ and $\zeta$ is continuous, by Morera's theorem $\zeta(z)$ is analytic in $R$.

To complete the discussion we must show that the limit function $\zeta(z)=U(z)+i V(z)$ of the chosen subsequence $\left\{f_{*}^{\left(h_{k}\right)}\right\}$ satisfies the given boundary condition $\zeta=\psi(s)$ on $C$, the boundary of $R$. It is sufficient for this purpose to consider the real-valued function $U=R \operatorname{e}\{\zeta\}$ and show that $U=\operatorname{Re}\{\psi(s)\}$ on $C$. Let $Q$ be a fixed point of $C$. By hypothesis we can construct a circle lying outside $C$ and intersecting $C$ only at the point $Q$, see Feller [4]. We denote the center of this circle by $A$, its radius by $\rho$, and let $P$ denote an arbitrary point of $R$ whose distance from $A$ is $r$.

For an arbitrary $\varepsilon>0$, we define the functions [4]

$$
U_{1}(P)=F(Q)+\varepsilon+K\left(\frac{1}{\rho}-\frac{1}{r}\right),
$$

and

$$
U_{2}(P)=F(Q)-\varepsilon-K\left(\frac{1}{\rho}-\frac{1}{r}\right),
$$

where $F=\operatorname{Re}\{\psi\}$ and $K$ is a positive constant to be determined later. On any semi-lattice

$$
\nabla U_{1}(P)=-K\left[r^{-3}+0(h)\right]<0,
$$




$$
\nabla U_{2}(P)>0
$$

in $R$ provided that $h$ is sufficiently small. Now if $u(P)$ is a solution of the differential-difference equation $\nabla u=0$ for the semi-lattice, by (3.3) the function $U_{1}(P)-u(P)$ is SD super-harmonic for $P \in R$. Accordingly, by Theorem 2.2, it assumes its minimum on $C$. Similarly, the function $U_{2}(P)-u(P)$ is $\mathrm{SD}$ sub-harmonic and by Theorem 2.1 assumes its maximum on $C$.

The argument given by Feller [4] now applies directly. We consequently establish that

$$
\varlimsup_{P \rightarrow Q} U(P) \leqq F(Q),
$$

and

$$
\lim _{\overline{p \rightarrow Q}} U(P) \geqq F(Q)
$$

which completes the proof.

\section{BIBLIOGRAPHY}

1. R. L. Helmbold, Semi-discrete potential theory, Carnegie Institute of Technology, Technical Report No. 34, Contract No. DA-36-061-ORD-490, Office of Ordnance Research, U. S. Army.

2. O. D. Kellogg, Foundations of Potential Theory, Dover Publications, Inc., New York (1953).

3. G. J. Kurowski, Semi-discrete analytic functions, Trans. Soc., 106, No. 1 (1963), 1-18. 4. J. D. Tamarkin and W. Feller, Partial Differential Equations, Brown University Notes, Providence, R.I., (1941). 



\section{PACIFIC JOURNAL OF MATHEMATICS}

\section{EDITORS}

Robert Osserman

Stanford University

Stanford, California

M. G. Arsove

University of Washington

Seattle 5 , Washington
J. DugundjI

University of Southern Califorma: Los Angeles 7, California

Lowell J. Paige

University of California

Los Angeles 24, California

\section{ASSOCIATE EDITORS}

E. F. BECKENBACH

B. H. NeumanN

F. WOLF

K. YosIDA

\section{SUPPORTING INSTITUTIONS}

UNIVERSITY OF BRITISH COLUMBIA

CALIFORNIA INSTITUTE OF TECHNOLOGY

UNIVERSITY OF CALIFORNIA

MONTANA STATE UNIVERSITY

UNIVERSITY OF NEVADA

NEW MEXICO STATE UNIVERSITY

OREGON STATE UNIVERSITY

UNIVERSITY OF OREGON

OSAKA UNIVERSITY

UNIVERSITY OF SOUTHERN CALIFORNIA
STANFORD UNIVERSITY

UNIVERSITY OF TOKYO

UNIVERSITY OF UTAH

WASHINGTON STATE UNIVERSITY

UNIVERSITY OF WASHINGTON

AMERICAN MATHEMATICAL SOCIETY CALIFORNIA RESEARCH CORPORATION SPACE TECHNOLOGY LABORATORIES NAVAL ORDNANCE TEST STATION 


\section{Pacific Journal of Mathematics}

\section{Vol. 14, No. 1 \\ May, 1964}

Richard Arens, Normal form for a Pfaffian .........................

Charles Vernon Coffman, Non-linear differential equations on cones in Banach

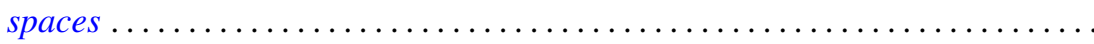

Ralph DeMarr, Order convergence in linear topological spaces ..............

Peter Larkin Duren, On the spectrum of a Toeplitz operator ................

Robert E. Edwards, Endomorphisms of function-spaces which leave stable all

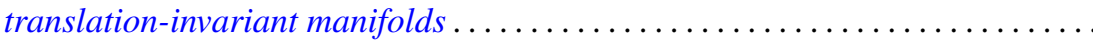

Erik Maurice Ellentuck, Infinite products of isols . . . . . . . . . . . . . . . . 49

William James Firey, Some applications of means of convex bodies . . . . . . . . 53

Haim Gaifman, Concerning measures on Boolean algebras ............. 61

Richard Carl Gilbert, Extremal spectral functions of a symmetric operator. . . . . . 75

Ronald Lewis Graham, On finite sums of reciprocals of distinct nth powers ..... 85

Hwa Suk Hahn, On the relative growth of differences of partition functions ...... 93

Isidore Isaac Hirschman, Jr., Extreme eigen values of Toeplitz forms associated

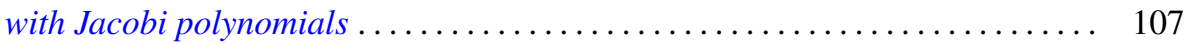

Chen-jung Hsu, Remarks on certain almost product spaces . . . . . . . . . . . 163

George Seth Innis, Jr., Some reproducing kernels for the unit disk . . . . . . . . . 177

Ronald Jacobowitz, Multiplicativity of the local Hilbert symbol . . . . . . . . . . . 187

Paul Joseph Kelly, On some mappings related to graphs ................. 191

William A. Kirk, On curvature of a metric space at a point . . . . . . . . . . . . 195

G. J. Kurowski, On the convergence of semi-discrete analytic functions . . . . . . . 199

Richard George Laatsch, Extensions of subadditive functions . . . . . . . . . . . 209

V. Marić, On some properties of solutions of $\Delta \psi+A\left(r^{2}\right) X \nabla \psi+C\left(r^{2}\right) \psi=0 \ldots 217$

William H. Mills, Polynomials with minimal value sets . . . . . . . . . . . 225

George James Minty, Jr., On the monotonicity of the gradient of a convex

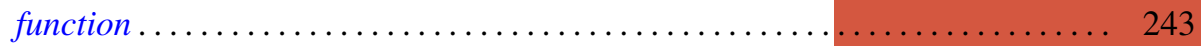

George James Minty, Jr., On the solvability of nonlinear functional equations of 'monotonic' type ................................... 249

J. B. Muskat, On the solvability of $x^{e} \equiv e(\bmod p) \ldots \ldots \ldots \ldots \ldots \ldots \ldots \ldots . \ldots \ldots$

Zeev Nehari, On an inequality of $P . R$. Bessack ................... 261

Raymond Moos Redheffer and Ernst Gabor Straus, Degenerate elliptic

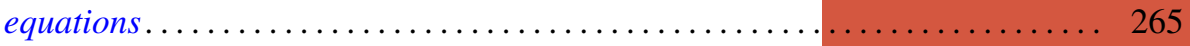

Abraham Robinson, On generalized limits and linear functionals . . . . . . . . . 269

Bernard W. Roos, On a class of singular second order differential equations with a



Tôru Saitô, Ordered completely regular semigroups . . . . . . . . . . . . . . . . 295

Edward Silverman, A problem of least area ....................... 309

Robert C. Sine, Spectral decomposition of a class of operators . . . . . . . . . 333

Jonathan Dean Swift, Chains and graphs of Ostrom planes . . . . . . . . . . . 353

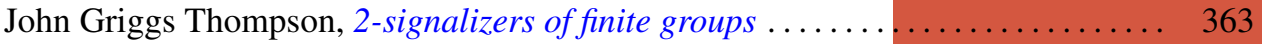

Harold Widom, On the spectrum of a Toeplitz operator . . . . . . . . . . . . . 365 